Supporting Information:

\title{
Spontaneous Symmetry Breaking of Nanoscale Spatiotemporal Pattern as the Origin of Helical Nanopore Etching in Silicon
}

Takumi Yasuda, ${ }^{\dagger}$ Yuki Maeda, ${ }^{\dagger}$ Kenta Matsuzaki, ${ }^{\dagger}$ Yutaka Okazaki,,$₫ \S$ Reiko Oda, ${ }^{\S}$

Atsushi Kitada ${ }^{\dagger}$ Kuniaki Murase,${ }^{\dagger}$ and Kazuhiro Fukami ${ }^{*} \uparrow$

${ }^{\dagger}$ Department of Materials Science and Engineering, Kyoto University, Kyoto 606-8501, Japan

$\$$ Graduate School of Energy Science, Kyoto University, Kyoto 606-8501, Japan

$\S$ Institute de Chimie et Biologie des Membranes \& des Nanoobjets (UMR5248 CBMN),

CNRS - Université de Bordeaux - Bordeaux INP, 33607 Pessac, France

*e-mail: fukami.kazuhiro.2u@kyoto-u.ac.jp 


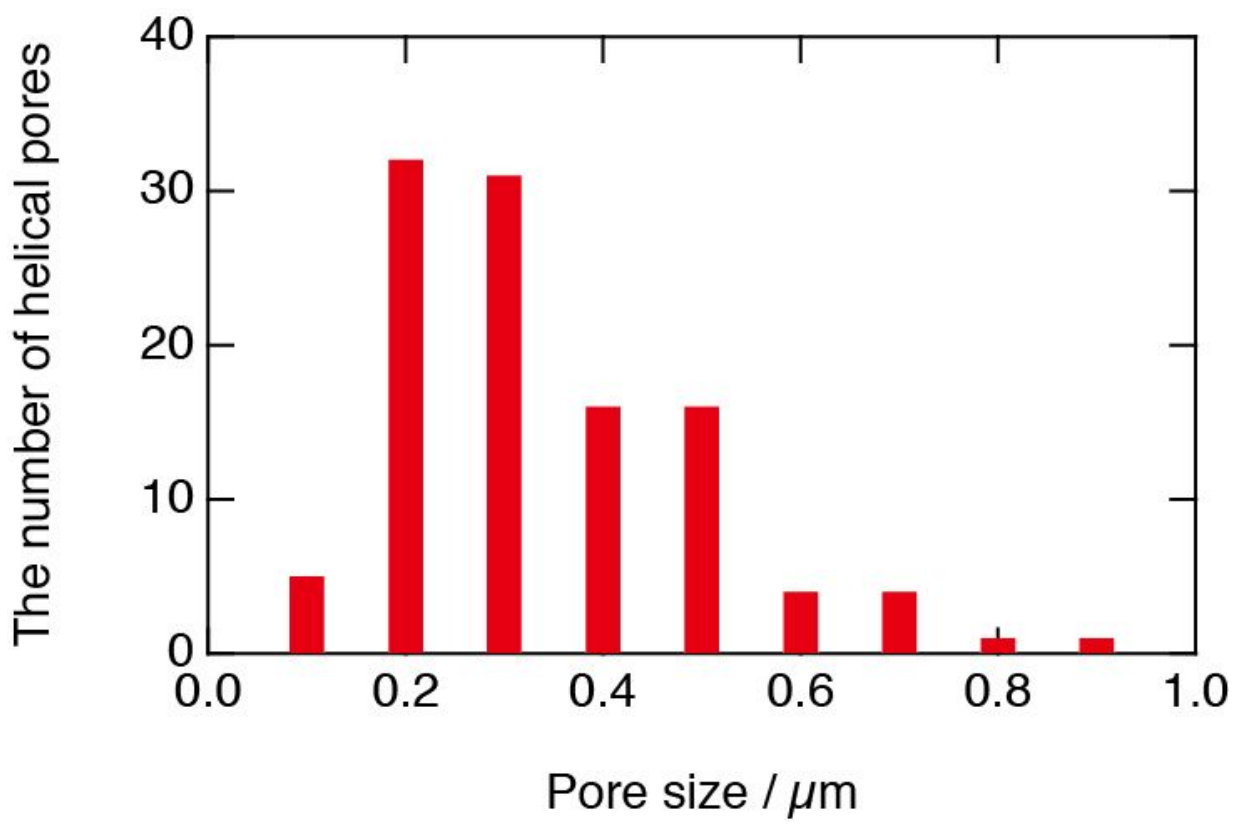

Figure S1. Histogram in pore size distribution of helical nanopore formed by PacEtch of silicon. 


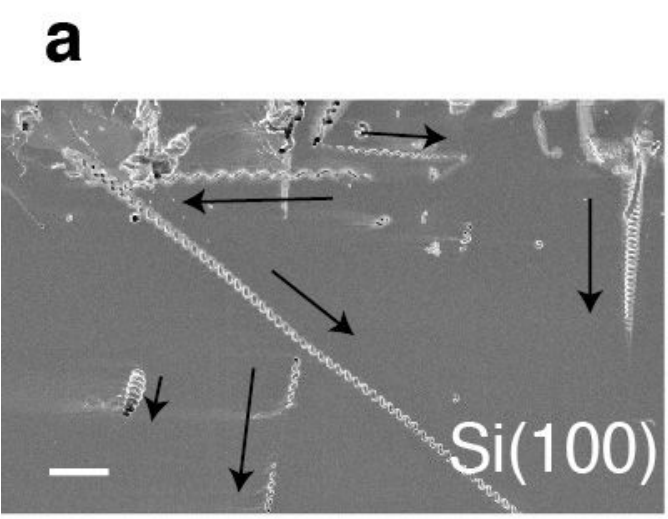

b

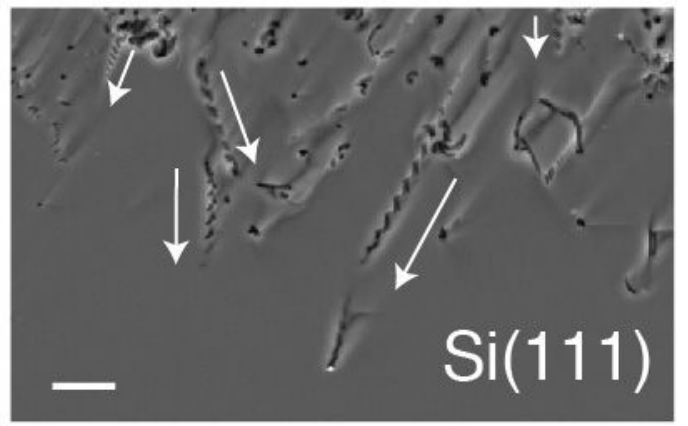

Figure S2. Cross-sectional images of silicon wafers after PacEtch. The orientation of the substrates was (a) $\mathrm{Si}(100)$ and (b) $\mathrm{Si}(111)$, respectively. The arrows in the figures indicate the direction of the axis of the helical nanopore. The arrows suggest that the direction of the helical nanopore growth is independent of the crystallographic orientation of silicon wafers. The scale bars indicate $5 \mu \mathrm{m}$. 


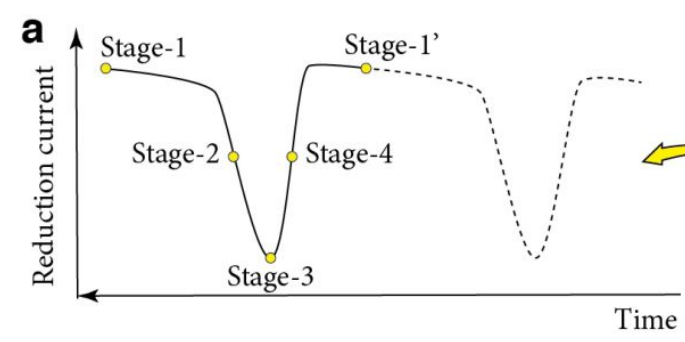

b

Oscillatory reduction wave at the interface

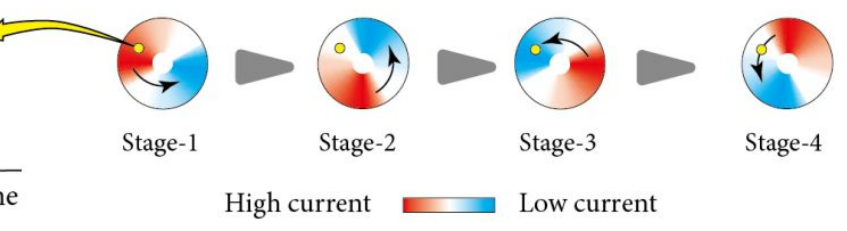

Figure S3. (a) Oscillatory reduction current for hydrogen peroxide measured on a platinum electrode. The oscillation is a sum of coherent local oscillators. (b) An example of non-uniform oscillatory wave propagation in a periodic manner. The total current must be constant; however, the oscillatory condition is satisfied locally. 


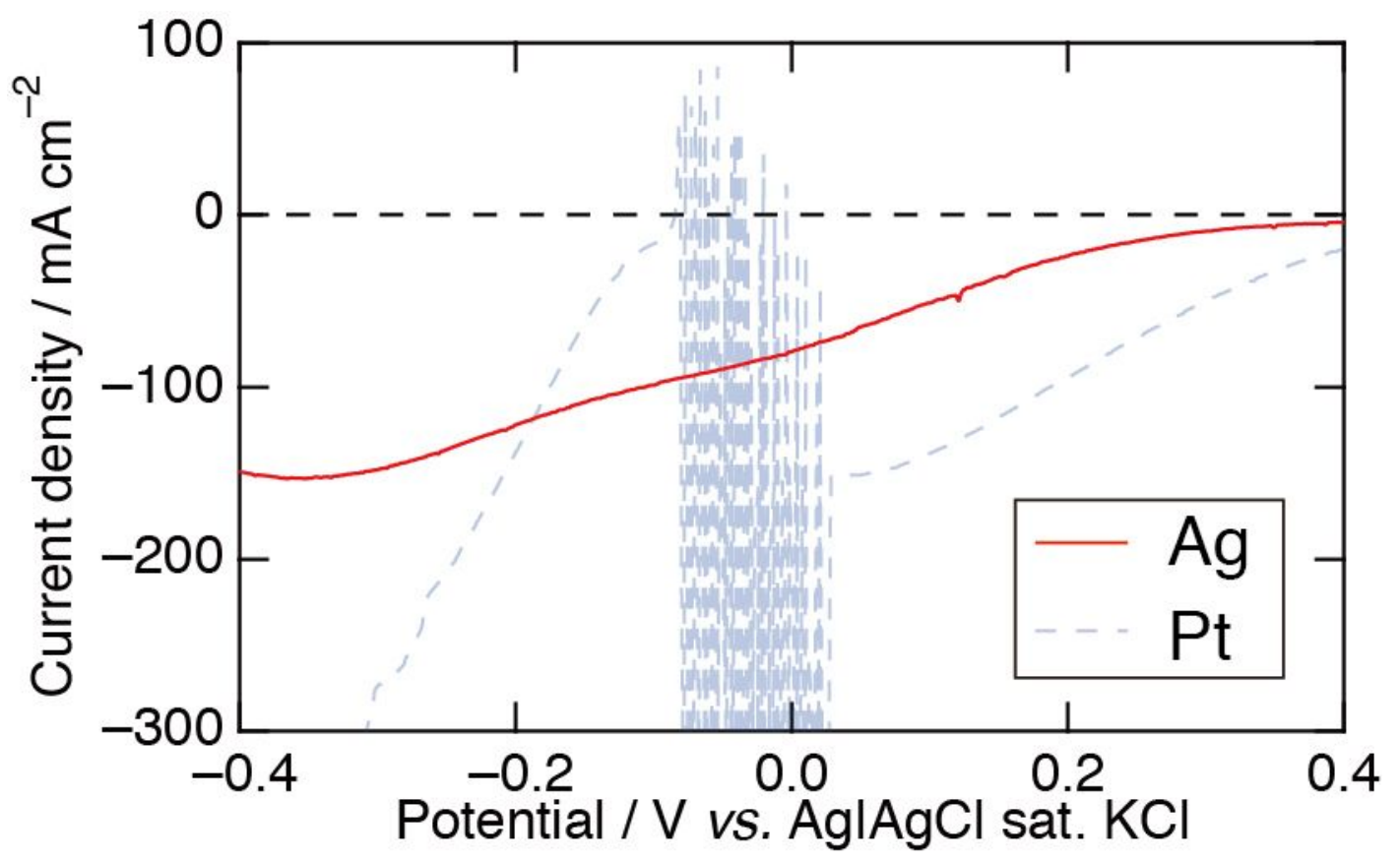

Figure S4. Current density vs. potential curve measured on a Ag electrode (red solid curve). A typical current density vs. potential curve measured on a Pt electrode is also plotted for comparison (gray dashed line). The curve was measured in the high HF concentration etchant ( $48 \mathrm{wt} \% \mathrm{HF}: 30 \mathrm{wt} \% \mathrm{H}_{2} \mathrm{O}_{2}=10: 1$ ) with the scan rate being $0.1 \mathrm{~V}$ $\mathrm{s}^{-1}$. 


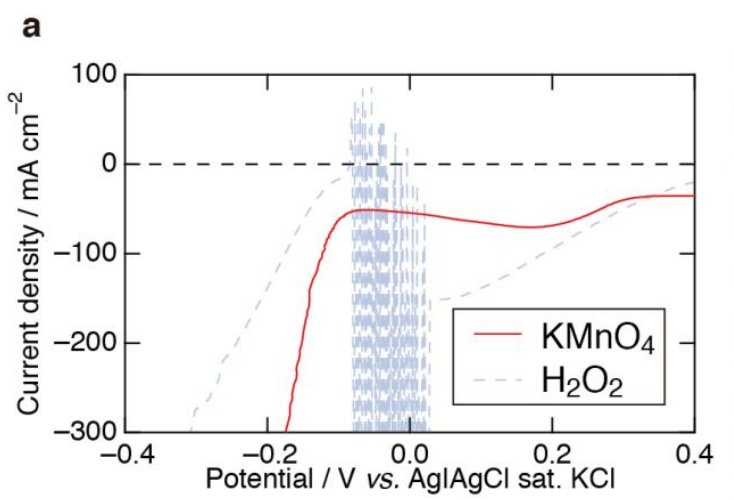

b

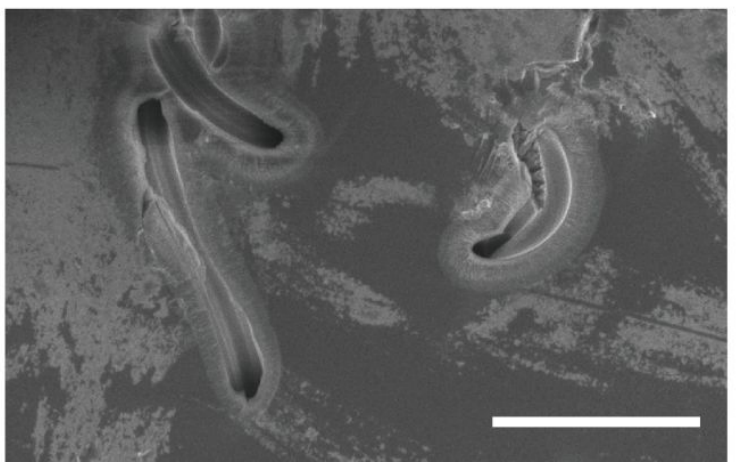

Figure S5. (a) Current density vs. potential curve measured on a Pt electrode in an etchant of $\mathrm{HF}+\mathrm{KMnO}_{4}$ (red solid curve) with the scan rate being $0.1 \mathrm{~V} \mathrm{~s}^{-1}$. A typical current density vs. potential curve measured on a Pt electrode in an etchant of HF + $\mathrm{H}_{2} \mathrm{O}_{2}$ was also shown for comparison (gray dashed line). (b) Cross-sectional SEM image of a silicon wafer after PacEtch in the etchant with $\mathrm{KMnO}_{4}$. The scale bar indicates $5 \mu \mathrm{m}$. 

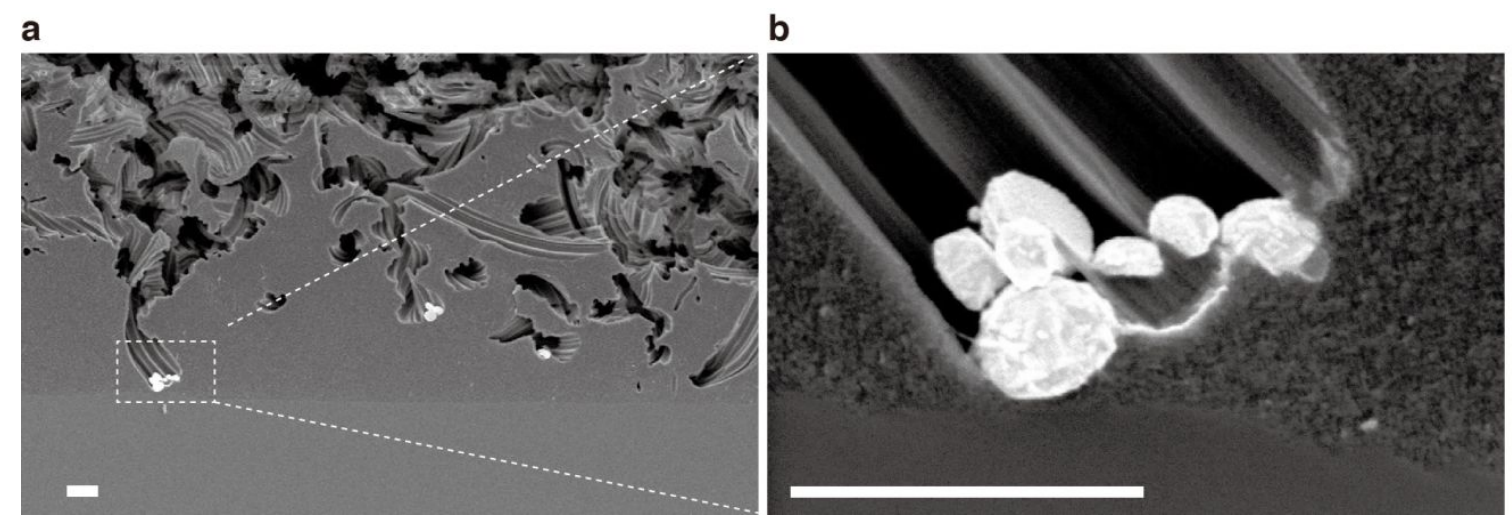

Figure S6. Cross-sectional SEM images of a "highly-doped" p-type silicon wafer with a resistivity of $0.005-0.03 \Omega \mathrm{cm}$ after PacEtch in the etchant of $\mathrm{HF}+\mathrm{H}_{2} \mathrm{O}_{2}$. (a) Low magnification image of the cross-sectional view. (b) High magnification image. Formation of microporous silicon is clearly observed. The scale bars indicate $1 \mu \mathrm{m}$. 


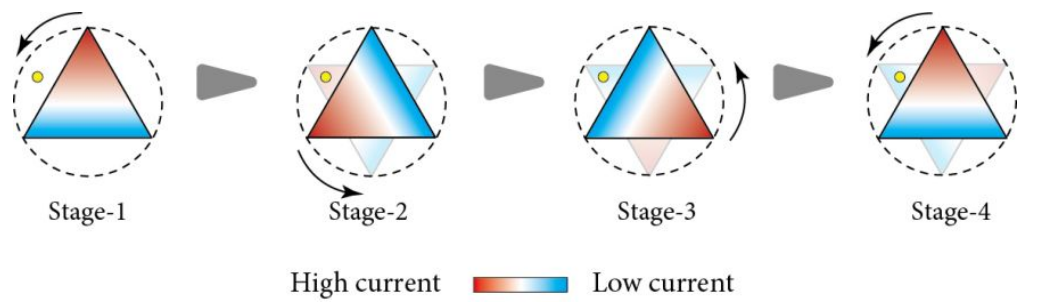

Figure S7. Illustration explaining that the motion of triangular nanoplate catalyst is not expected in the spatiotemporal pattern formation. The local site marked in yellow does not satisfy the oscillatory reaction with the rotation of the triangular nanoplate catalyst. Please see Stage-1 where the local site is located out of the interface between silicon and platinum; therefore, the reaction at the local site is discontinuous and the oscillatory reaction is not expected at the local site. 
(a)

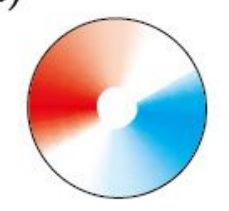

(b)

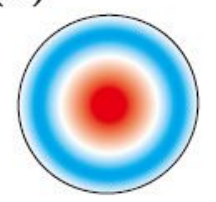

(c)

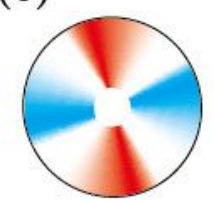

\section{High current}

\section{Low current}

Figure S8. Illustrations of the distribution of hydrogen peroxide reduction rate at the platinum/silicon interface when using a circular platinum catalyst that is almost perfectly symmetric. The case (b) seems to result in a metastable PacEtch. 